\title{
EXTRINSIC EIGENVALUES ESTIMATES FOR HYPERSURFACES IN PRODUCT SPACES
}

\author{
JULIEN ROTH
}

\begin{abstract}
We prove extrinsic upper bounds for the first eigenvalue of second order operator of divergence type as well as for Paneitz-like operators and two generalized Steklov problems on hypersurfaces of product spaces $N \times \mathbb{R}$. Examples of equality cases are given.
\end{abstract}

\section{Introduction AND STATEMENTS OF THE RESUlts}

In his seminal paper [18, Reilly proved the following well-known upper bound for the first non-zero eigenvalue of the Laplace operator on a closed $n$-dimensional submanifold $M$ of a Euclidean space $\mathbb{R}^{m}$

(1) ?Reilly1?

$$
\lambda_{1}(M) \leqslant \frac{n}{V(M)} \int_{M}\|H\|^{2} d v_{g}
$$

where $V(M)$ is the volume of $(M, g), d v_{g}$ its volume element and $H$ is the mean curvature vector of the isometric immersion of $(M, g)$ into $\mathbb{R}^{m}$. This inequality has been extended by many authors in different contexts: for other ambient spaces [15, 12], in terms of higher order mean curvatures [18], other operators [1, 2, 4, 14, 20, in the anisotropic setting [19, for weighted ambient spaces [5, 11, 20] or for differential forms [21] and spinors [3, 13. Recently, Xiong [23] obtained extrinsic estimates of Reilly type for closed hypersurfaces of product spaces $\left(\mathbb{R} \times N, d t^{2} \oplus h\right)$, where $\left(N^{n}, h\right)$ is a complete Riemannian manifold. In particular, he proved that the first eigenvalue $\lambda_{1}$ of the Laplace operator and the first eigenvalue $\sigma_{1}$ of the Steklov problem for mean-convex hypersurfaces (bounding a domain for the second one) satisfy respectively

$$
\lambda_{1} \leqslant n \kappa_{+}(M)\|H\|_{\infty} \quad \text { and } \quad \sigma_{1} \leqslant \kappa_{+}(M) \frac{\|H\|_{\infty}}{\inf _{M} H} .
$$

In the present note, we prove extrinsic eigenvalue estimates for four types of eigenvalues, namely for divergence-type operators $L_{T}$ (Theorem 1.1), Paneitz-like operators (Theorem 1.3), Steklov-Wentzell problem (Theorem 1.5) and biharmonic Steklov problem (Theorem 1.6). These results are extensions for product manifolds $\mathbb{R} \times N$ of the estimates obtained by the author in [20] for hypersurfaces of Euclidean spaces.

Date: July 2, 2019.

2010 Mathematics Subject Classification. 35P15, 58C40.

Key words and phrases. Reilly-type inequality, eigenvalue estimates, hypersurfaces, product manifolds. 
1.1. $L_{T}$ operators. Let $\left(M^{n}, g\right)$ be a closed connected and oriented Riemannian manifold and consider $T$ a symmetric, divergence-free and positive definite $(1,1)$ tensor over $M$. We associate with $T$ the following second order differential operator $L_{T}$ defined by $L_{T} f=-\operatorname{div}(\nabla f)$ for any $\mathcal{C}^{2}$ function on $M$, where $\operatorname{div}$ and $\nabla$ are respectively the divergence and the gradient over $\left(M^{n}, g\right)$. Under the above assumptions on $T$, the operator $L_{T}$ is self-adjoint, elliptic and positive. In particular, its spectrum is a increasing sequence of real numbers

$$
0=\lambda_{0}<\lambda_{1} \leqslant \cdots \leqslant \lambda_{k} \leqslant \longrightarrow+\infty .
$$

The eigenvalue 0 is simple and corresponds to constant eigenfunctions. In the sequel, we will consider the first positive eigenvalue $\lambda_{1}$. Now, assume that $\left(M^{n}, g\right)$ is isometrically immersed into a Riemannian product $\mathbb{R} \times N$. We set

$$
H_{T}=\operatorname{tr}(T S),
$$

where $S$ is the shape operator of the immersion.

For the well understanding of the statement of the result, we will introducre at this point the following notations: if $A$ is a $(1,1)$-tensor over $M$, then we denote

$$
A_{-}=\min \left\{A_{-}(x) \mid x \in M\right\}
$$

where $A_{-}(x)$ is the smallest eigenvalue of $A$ at the point $x$ and

$$
A_{+}=\max \left\{A_{+}(x) \mid x \in M\right\}
$$

where $A_{+}(x)$ is the biggest eigenvalue of $A$ at the point $x$.

Now, we can state the first result of this note which gives an extrinsic upper bound $\langle\operatorname{thm} 1\rangle$

for the first eigenvalue of $L_{T}$. Namely, we have the following

Theorem 1.1. Let $\left(N^{n}, h\right)$ be a complete Riemannian manifold and $\left(M^{n}, g\right)$ be a closed oriented Riemannian manifold isometrically immersed into the Riemannian product $\left(\mathbb{R} \times N, d t^{2} \oplus h\right)$. Moreover, let $T$ be a symmetric, positive definite and divergence-free (1,1)-tensor over $M$ and assume that $H_{T}$ is a positive function. Then, the first eigenvalue $\lambda_{1}$ of the operator $L_{T}$ on $M$ satisfies

$$
\lambda_{1} \leqslant \frac{(T S)_{+}}{T_{-}}\left\|H_{T}\right\|_{\infty} .
$$

Moreover, if $T$ and $S$ commute, then we have

$$
\lambda_{1} \leqslant \kappa_{+}(M)\left\|H_{T}\right\|_{\infty} .
$$

Remarks 1.2. (1) Note that since $H_{T}=\operatorname{tr}(T S)>0$, then $(T S)_{+}>0$ and the upper bound in the theorem is positive.

(2) We also want to point out that in the case where $T$ and $S$ commute, the hypotheses that $T$ is positive definite and $H_{T}$ is a positive function imply that $M$ has necessarily at least one positive principal curvature and so the upper bound $\kappa_{+}(M)\left\|H_{T}\right\|_{\infty}$ is positive.

(3) In particular $T$ and $S$ commute if $T$ is one the tensors $T_{r}$ associated with the higher order mean curvatures $H_{r}$. They will be considered in the first example of Section 3 . 
1.2. Paneitz-like operators. On a 4-dimensional Riemannian manifold $\left(M^{4}, g\right)$, the Paneitz operator, first introduced in [16] by Paneitz (see also [17]), is the fourth order differential operator $P$ defined by

$$
P u=\Delta^{2} u-\operatorname{div}\left(\frac{2}{3} \operatorname{Scal} \nabla u-2 \operatorname{Ric}(\nabla u)\right),
$$

for any $\mathcal{C}^{4}$ function $u$, where div is the divergence, $\Delta=-\operatorname{div} \nabla$ the Laplacian, Scal the scalar curvature and Ric the $(1,1)$-Ricci tensor associated with the metric $g$. It has been generalized in any dimension by Branson [6]. Namely, we have for $n \geqslant 5$,

$$
P u=\Delta^{2} u--\operatorname{div}\left(\frac{(n-2)^{2}+4}{2(n-1)(n-2)} \operatorname{Scal} \nabla u-\frac{4}{n-2} \operatorname{Ric}(\nabla u)\right)+\frac{n-4}{2} Q u,
$$

where $Q$ is the Branson $Q$-curvature associated with the metric $g$. The Paneitz operator is conformally covariant and plays a crucial role in the problem of prescribing $Q$-curvature. In the last two decades, the Paneitz operator (and its links with $Q$-curvature) has been intensively studied by many authors (see [10] and reference therein for instance).

Here, we are interesting in the spectrum of the Paneitz operator and more generally of Paneitz-like operators (for which the classical Paneitz operator in dimension 4 is a particular case). In 20] we obtain general Reilly-type upper bounds generalizing previous estimates proved by Chen and $\mathrm{Li}$ in $[8]$. The Paneitz-like operators are defined for some constants $a$ and $b$ with $b \geqslant-\frac{n}{n-1}$ by

$$
P_{a, b} u=\Delta^{2} u-\operatorname{div}(a \operatorname{Scal} \nabla u+b \operatorname{Ric} \nabla u),
$$

for any smooth function $u$ on $M$. The fourth order operator $P_{a, b}$ is elliptic and self-adjoint so that it has a discrete real spectrum. In the sequel, we will restrict to the case where $P_{a, b}$ is positive. The positivity of $P_{a, b}$ is ensured under some curvature lower bounds (see 24] for more details). Here, we give upper bounds for the first eigenvalue of $P_{a, b}$ for hypersurfaces in products spaces $\mathbb{R} \times N$. Namely, we $\langle$ thm2〉 prove the following

Theorem 1.3. Let $\left(N^{n}, h\right)$ be a complete Riemannian manifold and $\left(M^{n}, g\right)$ be a closed oriented Riemannian manifold isometrically immersed into the Riemannian product $\left(\mathbb{R} \times N, d t^{2} \oplus h\right)$. Let $a$ and $b$ two real constants with $b \geqslant-\frac{n}{n-1}$ and $n a+b \geqslant 0$. Moreover, assume that $M$ has nonnegative scalar curvature and that the Paneitz-like operator $P_{a, b}$ is positive. Then, the first eigenvalue $\Lambda_{1}$ of the Paneitzlike operator $P_{a, b}$ on $M$ satisfies

$$
\Lambda_{1} \leqslant n \kappa_{+}(M)\|H\|_{\infty}\left(n \kappa_{+}(M)\|H\|_{\infty}+(a \mathrm{Scal} \mathrm{Id}+b \mathrm{Ric})_{+}\right) .
$$

Remark 1.4. This result is of interest only if the operator $P_{a, b}$ is positive. As mentioned, see 24] for details about the positivity of $P_{a, b}$.

1.3. Steklov-Wentzell problem. Let $\Omega$ be a smooth domain of the Riemannian product $\mathbb{R} \times N$ with non-empty boundary $M=\partial \Omega$ and $b$ a nonnegative constant. We will denote by $g$ the induced metric on $M$ and denote by $\bar{\Delta}$ and $\Delta$ the Laplacian on $\Omega$ and $M$ respectively. We consider the following Steklov-type problem for the 
Laplacian $\bar{\Delta}$ with the so-called Wentzell boundary condition. Namely, we consider

(SW) ? Wentzell?

$$
\begin{cases}\bar{\Delta} f=0 & \text { on } \Omega \\ -b \Delta f-\frac{\partial f}{\partial \nu}=\alpha f & \text { on } M .\end{cases}
$$

where $\frac{\partial f}{\partial \nu}=<\bar{\nabla} f, \nu>$ is the derivative of the function $f$ with the respect to the inner unit normal $\nu$. Here, $\bar{\nabla}$ is the gradient over $\Omega$. Note that, if $b=0$, then, we recover the classical Steklov problem. The spectrum of this problem is an increasing sequence (see 9, 22])

$$
0=\alpha_{0}<\alpha_{1} \leqslant \alpha_{2} \cdots \leqslant \alpha_{k} \leqslant \cdots \longrightarrow+\infty \text {. }
$$

The eigenvalue 0 is simple and the corresponding eigeinfunctions are the constant ones. Here again, we prove a Reilly-type upper bound for the first positive eigen$\langle\operatorname{thm} 3\rangle$ value of this problem. Namely, we have

Theorem 1.5. Let $\left(N^{n}, h\right)$ be a complete Riemannian manifold and $\left(M^{n}, g\right)$ be a closed oriented Riemannian manifold isometrically immersed into the Riemannian product $\left(\mathbb{R} \times N, d t^{2} \oplus h\right)$. Moreover, assume that $M$ is mean-convex and bounds a domain $\Omega$ in $\mathbb{R} \times N$. Then, the first eigenvalue $\alpha_{1}$ of the Steklov-Wentzell problem satisfies

$$
\alpha_{1} \leqslant \kappa_{+}(M)\|H\|_{\infty}\left(\frac{1}{\inf _{M} H}+b n\right) .
$$

1.4. Biharmonic Steklov problem. Let $\Omega$ be a smooth domain of the riemannian product $\mathbb{R} \times N$ with non-empty boundary $M=\partial \Omega$ and $\tau$ a positive constant. We consider the following biharmonic Steklov problem.

$$
\left(\begin{array}{ll}
\bar{\Delta}^{2} f-\tau \bar{\Delta} f=0 & \text { on } \Omega, \\
\operatorname{Steklovbsh}^{\frac{\partial^{2} f}{\partial \nu^{2}}=0} & \text { on } M, \\
\tau \frac{\partial f}{\partial \nu}-\operatorname{div}_{\partial M}\left(P_{\partial M}\left(\left(\nabla^{2} f\right) \nu\right)\right)-\frac{\partial \Delta f}{\partial \nu}=\beta f & \text { on } M .
\end{array}\right.
$$

where $P_{\partial M}$ is the projection over the tangent space of $\partial M$. This problem has a discret spectrum consisting in an increasing sequence (see [7])

$$
0=\beta_{0}<\beta_{1} \leqslant \beta_{2} \cdots \leqslant \beta_{k} \leqslant \cdots \longrightarrow+\infty .
$$

The eigenvalue 0 is simple and the corresponding eigeinfunctions are the constant functions. In the next theorem, we prove an extrinsic upper for the first positive $\langle\operatorname{thm} 4\rangle$ eigenvalue of this problem.

Theorem 1.6. Let $\left(N^{n}, h\right)$ be a complete Riemannian manifold and $\left(M^{n}, g\right)$ be a closed oriented Riemannian manifold isometrically immersed into the Riemannian product $\left(\mathbb{R} \times N, d t^{2} \oplus h\right)$. Moreover, assume that $M$ is mean-convex and bounds a domain $\Omega$ in $\mathbb{R} \times N$. Then, the first eigenvalue of the biharmonic Steklov problem satisfies

$$
\beta_{1} \leqslant \tau \kappa_{+}(M) \frac{\|H\|_{\infty}}{\inf _{M} H}
$$


After giving the proof of these four theorems in Section 2, we will give some examples of their equality cases in Section 3 .

\section{$\langle\sec 2\rangle$}

\section{Proofs of the Results}

2.1. Proof of Theorem 1.1. We recall that the variational characterization of $\lambda_{1}$ given by

$$
\lambda_{1}=\inf \left\{\frac{\int_{M}\langle T \nabla f, \nabla f\rangle d v_{g}}{\int_{M} f^{2} d v_{g}} \mid u \neq 0, \int_{M} f d v_{g}=0\right\} .
$$

Here, $\nabla u$ stands for the gradient of the function $u$ over $M$ and $d v_{g}$ is the Riemannian volume form of $M$. Note that in the sequel, we will also use, without confusion, $\nabla$ for the Levi-Civita connection of $(M, g)$.

We will use as test function the function $t$ which is the coordinate in the factor $\mathbb{R}$ of the product $\mathbb{R} \times N$. First, obviously, $M$ is invariant by translation in the direction of $\mathbb{R}$, so we can assume that $\int_{M} t d v_{g}=0$. Second, since the function $H_{T}$ is positive, we deduce that $t$ does not vanish identically. Indeed, if $t$ vanishes identically over $M$, then $M$ is included in the slice $\{0\} \times N$. Since $M$ is a closed manifold, this is possible if and only if $M=N$ and so $M$ is totally geodesic in the product $N \times \mathbb{R}$. This is a contradiction with the fact that $H_{T}>0$. Hence, $t$ does not vanish identically and can be used as a test function. Thus, we have

$$
\lambda_{1} \leqslant \frac{\int_{M}\langle T(\nabla t), \nabla t\rangle d v_{g}}{\int_{M} t^{2} d v_{g}} .
$$

Now, let us compute $L_{T} t$. For more convience, let $p \in M$ and consider $\left\{e_{1}, \cdots, e_{n}\right\}$ be a normal frame at $p$. We have

$$
\begin{aligned}
L_{T} t & =-\operatorname{div}(T \nabla t) \\
& =-\sum_{i=1}^{n}\left\langle\nabla_{e_{i}}(T \nabla t), e_{i}\right\rangle \\
& =-\sum_{i, j=1}^{n}\left\langle\nabla_{e_{i}}\left(\left\langle\nabla t, e_{j}\right\rangle T e_{j}\right), e_{i}\right\rangle \\
& \left.=-\sum_{i, j=1}^{n} e_{i}\left(\left\langle\nabla t, e_{j}\right) T e_{j}, e_{i}\right\rangle+\sum_{i, j=1}^{n}\left\langle\nabla t, e_{j}\right\rangle \nabla_{e_{i}}\left(T e_{j}\right), e_{i}\right\rangle \\
& =-\sum_{i, j=1}^{n} e_{i}\left(\left\langle\nabla t, e_{j}\right) T e_{j}, e_{i}\right\rangle,
\end{aligned}
$$

where the second part of the right hand side vanishes in the last line since $T$ is divergence free. Hence, denoting by $\bar{\nabla}$ the Levi-Civita connection of $\left(\mathbb{R} \times N, d t^{2} \oplus h\right)$, 
and $T_{i, j}=\left\langle T e_{i}, e_{j}\right\rangle$, we have

$$
\begin{aligned}
L_{T} t & =-\sum_{i, j=1}^{n}\left\langle\bar{\nabla}_{e_{i}} \nabla t, e_{j}\right\rangle T_{i, j} \\
& =-\sum_{i, j=1}^{n}\left\langle\bar{\nabla}_{e_{i}}\left(\partial_{t}-\left\langle\partial_{t}, \nu\right\rangle \nu\right), e_{j}\right\rangle T_{i, j},
\end{aligned}
$$

where $\nu$ is a unit normal vector field. Moreover, since $\partial_{t}$ is parallel for $\bar{\nabla}$ and $-\bar{\nabla}_{(\cdot)} \nu$ is the shape operator $S$, we get

$$
\begin{aligned}
L_{T} t & =-\sum_{i, j=1}^{n}\left\langle\partial_{t}, \nu\right\rangle\left\langle S e_{i}, e_{j}\right\rangle T_{i, j} \\
& =-\sum_{i=1}^{n}\left\langle\partial_{t}, \nu\right\rangle\left\langle S e_{i}, T e_{i}\right\rangle \\
& =-H_{T} u,
\end{aligned}
$$

where we have set $u=\left\langle\partial_{t}, \nu\right\rangle$. Then, we have

$$
\begin{aligned}
\lambda_{1} & \leqslant \frac{\int_{M} t L_{T} t d v_{g}}{\int_{M} t^{2} d v_{g}} \\
& \leqslant \frac{\left(\int_{M} t L_{T} t d v_{g}\right)^{2}}{\left(\int_{M} t^{2} d v_{g}\right)\left(\int_{M}\langle T \nabla t, \nabla t\rangle d v_{g}\right)}
\end{aligned}
$$

But, since $L_{T} t=-H_{T} u$, we have from the Cauchy-Schwarz inequality

$$
\left(\int_{M} t L_{T} t d v_{g}\right)^{2} \leqslant\left(\int_{M} H_{T}^{2} u^{2} d v_{g}\right)\left(\int_{M} t^{2} d v_{g}\right)
$$

and so

$$
\lambda_{1} \leqslant \frac{\int H_{T}^{2} d v_{g}}{\int_{M}\langle T \nabla t, \nabla t\rangle d v_{g}} .
$$

On the other hand, we have $u L_{T} t=-H_{T} u^{2}$, which after integration gives

(3) intHT $\int_{M} H_{T} u^{2} d v_{g}=-\int_{M}\langle T \nabla u, \nabla t\rangle d v_{g}=\int_{M}\langle T S \nabla t, \nabla t\rangle d v_{g}$,

since $\nabla u=\sum_{i=1}^{n} e_{i}(u) e_{i}=\sum_{i=1}^{n} e_{i}(\langle\nu, \partial t\rangle) e_{i}=-\sum_{i=1}^{n}\left\langle S e_{i}, \partial_{t}\right\rangle e_{i}=-S(\nabla t)$.

FInally, we get

$$
\lambda_{1} \leqslant\left\|H_{T}\right\|_{\infty} \frac{\int_{M}\langle T S \nabla t, \nabla t\rangle d v_{g}}{\int_{M}\langle T \nabla t, \nabla t\rangle d v_{g}}
$$


and so

$$
\lambda_{1} \leqslant \frac{(T S)_{+}}{T_{-}}\left\|H_{T}\right\|_{\infty} .
$$

We recall that $T_{-}=\min \left\{T_{-}(x) \mid x \in M\right\}$ where $T_{-}(x)$ is the smallest eigenvalue of $T$ at the point $x$. Note that $T_{-}$is a positive number since $T$ is positive definite and $M$ is compact. Also, $(T S)_{+}=\max \left\{(T S)_{+}(x) \mid x \in M\right\}$ where $(T S)_{+}(x)$ is the largest eigenvalue of $T S$ at the point $x$. Since $H_{T}$ is a positive function, $(T S)_{+}$is also a positive number.

Now, assume that $T$ and $S$ commute. Since $T$ is positive definite and symmetric, there exists a square root of $T$, denoted $U$ which is also symmetric, positive definite and which also commutes with $S$. Hence, we have

$$
\begin{aligned}
\lambda_{1} & \leqslant\left\|H_{T}\right\|_{\infty} \frac{\int_{M}\langle T S \nabla t, \nabla t\rangle d v_{g}}{\int_{M}\langle T \nabla t, \nabla t\rangle d v_{g}} \\
& \leqslant\left\|H_{T}\right\|_{\infty} \frac{\int_{M}\langle S U \nabla t, U \nabla t\rangle d v_{g}}{\int_{M}\langle U \nabla t, U \nabla t\rangle d v_{g}} \\
& \leqslant\left\|H_{T}\right\|_{\infty} \kappa_{+}(M) .
\end{aligned}
$$

This concludes the proof.

2.2. Proof of Theorem 1.3. From the variational characterization of $\Lambda_{1}$, we obtain, using $t$ as test function

$$
\begin{aligned}
\Lambda_{1} \int_{M} t^{2} d v_{g} & \leqslant \int_{M} t P_{a, b} t d v_{g} \\
& \leqslant \int_{M}\left(t \Delta^{2} t-t \operatorname{div}(a \operatorname{Scal} \nabla t+b \operatorname{Ric}(\nabla t))\right) d v_{g} \\
& \leqslant \int_{M}\left(|\Delta t|^{2}+a \operatorname{Scal}|\nabla t|^{2}+b\langle\operatorname{Ric}(\nabla t), \nabla t\rangle\right) d v_{g} \\
& \leqslant \int_{M}\left(|\Delta t|^{2}+(a \operatorname{Scal}+b \operatorname{Ric})_{+}\|\nabla t\|^{2}\right) d v_{g} .
\end{aligned}
$$

Note that from the assumption $n a+b \geqslant 0$ and Scal $\geqslant 0$, then $(a \mathrm{Scal}+b \mathrm{Ric})_{+}$is nonnegative. Moreover, as we have seen in the proof of Theorem 1.1 (with $T=\mathrm{Id}$ ), we have $\Delta t=-n H u$, with $u=\left\langle\partial_{t}, \nu\right\rangle$. Hence, we get

(4)

$$
\begin{aligned}
& \Lambda_{1} \leqslant \frac{n^{2} \int_{M} H^{2} u^{2} d v_{g}}{\int_{M} t^{2} d v_{g}}+(a \mathrm{Scal}+b \mathrm{Ric})_{+} \frac{\int_{M}\|\nabla t\|^{2} d v_{g}}{\int_{M} t^{2} d v_{g}} \\
& \text { (4) inegLambdaf iral }\left(\frac{n^{2} \int_{M} H^{2} u^{2} d v_{g}}{\int_{M}\|\nabla t\|^{2} d v_{g}}+(a \mathrm{Scal}+b \mathrm{Ric})+\right) \frac{\int_{M}\|\nabla t\|^{2} d v_{g}}{\int_{M} t^{2} d v_{g}} \text {. }
\end{aligned}
$$


First, since $t \Delta t=-n H u t$, we have

(5) Hut

$$
\begin{aligned}
\int_{M}\|\nabla t\|^{2} d v_{g} & =n \int_{M} H u t d v_{g} \\
& \leqslant n\left(\int_{M} H^{2} u^{2} d v_{g}\right)^{\frac{1}{2}}\left(\int_{M} t^{2} d v_{g}\right)^{\frac{1}{2}}
\end{aligned}
$$

by the Cauchy-Schwarz inequality. Hence, we get

$$
\begin{aligned}
\frac{\int_{M}\|\nabla t\|^{2} d v_{g}}{\int_{M} t^{2} d v_{g}} & \leqslant \frac{n^{2} \int_{M} H^{2} u^{2} d v_{g}}{\int_{M}\|\nabla t\|^{2} d v_{g}} \\
& \leqslant n\|H\|_{\infty} \frac{n \int_{M} H u^{2} d v_{g}}{\int_{M}\|\nabla t\|^{2} d v_{g}} \\
& \leqslant n\|H\|_{\infty} \frac{\int_{M}\langle S \nabla t, \nabla t\rangle d v_{g}}{\int_{M}\|\nabla t\|^{2} d v_{g}} \\
& \leqslant n\|H\|_{\infty} \kappa_{+}(M)
\end{aligned}
$$

where we have used (3) with $T=\mathrm{Id}$. Thus, reporting in (4), we obtain

$$
\Lambda_{1} \leqslant n \kappa_{+}(M)\|H\|_{\infty}\left(n \kappa_{+}(M)\|H\|_{\infty}+(a S \mathrm{Id}+b \mathrm{Ric})_{+}\right),
$$

which concludes the proof of Theorem 1.3

2.3. Proof of Theorem 1.5. First, we recall that the first eigenvalue $\alpha_{1}$ of Steklov-Wentzell problem has the following variational characterization (see [9, 22])$$
\underline{\text { scharacadphat }} \inf \left\{\frac{\int_{\Omega}\|\bar{\nabla} f\|^{2} d v_{\bar{g}}+b \int_{M}\|\nabla f\|^{2} d v_{g}}{\int_{M} f^{2} d v_{g}} \mid \int_{\partial M} f d v_{g}=0\right\} \text {. }
$$

As in the proof of Theorem 1.1, we may assume that the function $t$ satisfies $\int_{\partial M} t d v_{g}=0$ and thus use it as a test function. So, we get

$$
\alpha_{1} \leqslant \frac{\int_{\Omega}\|\bar{\nabla} t\|^{2} d v_{\bar{g}}}{\int_{M} t^{2} d v_{g}}+b \frac{\int_{M}\|\nabla t\|^{2} d v_{g}}{\int_{M} t^{2} d v_{g}} .
$$

First, we have

$$
\int_{\Omega}\|\bar{\nabla} t\|^{2} d v_{\bar{g}}=-\int_{\Omega} t \bar{\Delta} t d v_{\bar{g}}+\int_{\Omega} \operatorname{div}_{\bar{g}}(t \bar{\nabla} t) d v_{\bar{g}}
$$

Since $\bar{\Delta} t=0$, using the Stokes theorem, we get

$$
\int_{\Omega}\|\bar{\nabla} t\|^{2} d v_{\bar{g}}=\int_{M}\langle t \bar{\nabla} t, \nu\rangle d v_{g}=\int_{M} t u d v_{g}
$$


where $u$ is defined as above by $u=\left\langle\partial_{t}, \nu\right\rangle=\langle\bar{\nabla} t, \nu\rangle$. Hence, by the Cauchy-Schwarz inequality, we obtain

$$
\int_{\Omega}\|\bar{\nabla} t\|^{2} d v_{\bar{g}} \leqslant\left(\int_{M} t^{2} d v_{g}\right)^{\frac{1}{2}}\left(\int_{M} u^{2} d v_{g}\right)^{\frac{1}{2}}
$$

and thus

$$
\frac{\int_{\Omega}\|\bar{\nabla} t\|^{2} d v_{\bar{g}}}{\int_{M} t^{2} d v_{g}} \leqslant \frac{\left(\int_{M} u^{2} d v_{g}\right)^{\frac{1}{2}}}{\left(\int_{M} t^{2} d v_{g}\right)^{\frac{1}{2}}} .
$$

On the other hand, we have

$$
\begin{aligned}
n \inf _{M}(H) \int_{M} u^{2} d v_{g} & \leqslant \int_{M} n H u^{2} d v_{g} \\
& \leqslant \int_{M}\langle S \nabla t, \nabla t\rangle d v_{g} \\
& \leqslant \kappa_{+}(M) \int_{M}\|\nabla t\|^{2} d v_{g} \\
& \leqslant \kappa_{+}(M) \int_{M} n H u t d v_{g} \\
& \leqslant n \kappa_{+}(M)\|H\|_{\infty} \int_{M} u t d v_{g} \\
& \leqslant n \kappa_{+}(M)\|H\|_{\infty}\left(\int_{M} t^{2} d v_{g}\right)^{\frac{1}{2}}\left(\int_{M} u^{2} d v_{g}\right)^{\frac{1}{2}}
\end{aligned}
$$

where we have used (3) with $T=$ Id and (5) successively. Finally, we get

$$
\frac{\int_{\Omega}\|\bar{\nabla} t\|^{2} d v_{\bar{g}}}{\int_{M} t^{2} d v_{g}} \leqslant \frac{\kappa_{+}(M)\|H\|_{\infty}}{\inf _{M}(H)} .
$$

Moreover, proceeding as in the proof of Theorem 1.1 with $T=\mathrm{Id}$, we obtain immediately that

which gives finally

$$
\frac{\int_{M}\|\nabla t\|^{2} d v_{g}}{\int_{M} t^{2} d v_{g}} \leqslant n \kappa_{+}\|H\|_{\infty}
$$

$$
\alpha_{1} \leqslant \kappa_{+}(M)\|H\|_{\infty}\left(\frac{1}{\inf _{M} H}+b n\right) .
$$

2.4. Proof of Theorem 1.6. The boundary conditions in the biharmonic Steklov problem (BS) are the natural one so that the weak formulation of this problem is the following (see [7]):

$$
\int_{\Omega}\left(\left\langle\bar{\nabla}^{2} f, \bar{\nabla}^{2} \phi\right\rangle+\tau\langle\bar{\nabla} f, \bar{\nabla} \phi\rangle\right) d v_{\bar{g}}=\beta \int_{M} f \phi d v_{g}
$$


Hence, the first positive eigenvalue $\beta_{1}$ has the following variational characterization

(8) ?characbet $\beta_{1} ?=\inf \left\{\frac{\int_{\Omega}\left(\left\|\bar{\nabla}^{2} f\right\|^{2}+\tau\|\bar{\nabla} f\|^{2}\right) d v_{\bar{g}}}{\int_{M} f^{2} d v_{g}} \mid \int_{M} f d v_{g}=0\right\}$.

As previously, up to a possible translation, we use $t$ as test function in the above variational characterization so that

$$
\begin{aligned}
\beta_{1} \int_{M} t^{2} d v_{g} & \leqslant \int_{\Omega}\left(\left\|\bar{\nabla}^{2} t\right\|^{2}+\tau\|\bar{\nabla} t\|^{2}\right) d v_{\bar{g}} \\
& \leqslant \tau \int_{\Omega}\|\bar{\nabla} t\|^{2} d v_{\bar{g}}
\end{aligned}
$$

since $\bar{\nabla}^{2} t=0$. Thus, we have

$$
\beta_{1} \leqslant \tau \frac{\int_{\Omega}\|\bar{\nabla} t\|^{2} d v_{\bar{g}}}{\int_{M} t^{2} d v_{g}}
$$

From the proof of Theorem 1.5, we have (7)

$$
\frac{\int_{\Omega}\|\bar{\nabla} t\|^{2} d v_{\bar{g}}}{\int_{M} t^{2} d v_{g}} \leqslant \frac{\kappa_{+}(M)\|H\|_{\infty}}{\inf _{M}(H)}
$$

which concludes the proof of Theorem 1.6

\section{EXAmples of EQUALity CASE}

$\langle\sec 3\rangle$

We finish this note by giving examples of equality cases for each of the four theorems.

Example 3.1. We consider here the well-known operators $L_{r}$ associated to the higher order mean curvatures. The higher order mean curvatures are extrinsic quantities defined from the second fundamental form and generalizing the notion of mean curvature. Up to a normalisation constant the mean curvature $H$ is the trace of the second fundamental form $B$ :

$$
H=\frac{1}{n} \operatorname{tr}(B)
$$

In other words the mean curvature is

$$
H=\frac{1}{n} S_{1}\left(\kappa_{1}, \ldots, \kappa_{n}\right)
$$

where $S_{1}$ is the first elementary symmetric polynomial and $\kappa_{1}, \ldots, \kappa_{n}$ are the principal curvatures. Higher order mean curvatures are defined in a similar way for $r \in\{1, \ldots, n\}$ by

$$
H_{r}=\frac{1}{\left(\begin{array}{c}
n \\
r
\end{array}\right)} S_{r}\left(\kappa_{1}, \cdots, \kappa_{n}\right)
$$

where $S_{r}$ is the $r$-th elementary symmetric polynomial, that is for any $n$-tuple $\left(x_{1}, \cdots, x_{n}\right)$,

$$
S_{r}\left(x_{1}, \ldots, x_{n}\right)=\sum_{1 \leqslant i_{1}<\cdots<i_{r} \leqslant n} x_{i_{1}} \cdots x_{i_{r}} .
$$


By convention we set $H_{0}=1$ and $H_{n+1}=0$. Finally, for convenience we also set $H_{-1}=-\langle X, \nu\rangle$.

To each $H_{r}$ we associate a symmetric $(2,0)$-tensor, which is in coordinates given by

$$
T_{r}=\left(T_{r}^{i j}\right)=\left(\frac{\partial S_{r+1}}{\partial B_{i j}}\right)
$$

where $S_{r+1}$ is now understood to depend on the second fundamental form and the metric. The relation between these two notions can be found in 4 for example. These tensors $T_{r}$ are divergence-free (see [14] for instance) and satisfy the following relations:

$$
\operatorname{tr}\left(T_{r}\right)=c(r) H_{r} \quad \text { and } \quad H_{T_{r}}=-c(r) H_{r+1} \nu
$$

where $c(r)=(n-r)\left(\begin{array}{l}n \\ r\end{array}\right)$ and $H_{T_{r}}$ is given by the relation 2 . The operator $L_{r}$ is defined as the operator $L_{T_{r}}$ associated with the tensor $T_{r}$. Note that in space forms, if $H_{r+1}>0$, then $L_{r}$ is a positive operator (see [4]).

Now, we consider the sphere $\mathbb{S}^{n}(R)$ of radius $R$ into $\mathbb{R}^{n+1}=\mathbb{R} \times \mathbb{R}^{n}$. The first eigenvalue of $L_{r}$ is $\frac{c(r)}{R^{n+2}}$ (see [1]). On the other hand, since $T_{r}$ and $B$ commute, the bound of Theorem 1.1 is

$$
\kappa_{+}(M)\left\|H_{T_{r}}\right\|_{\infty}^{2}=\frac{c(r)}{R^{r+2}}
$$

since all the principal curvature are $\frac{1}{R}$ and $\left\|H_{T_{r}}\right\|=c(r) H_{r+1}=\frac{c(r)}{R^{r+1}}$. Hence equality occurs in Theorem 1.1 .

Example 3.2. Now, we consider the sphere $\mathbb{S}^{4}(R)$ into $\mathbb{R}^{5}=\mathbb{R} \times \mathbb{R}^{4}$ and $P=P_{\frac{2}{3}},-2$ the Paneitz operator on $\mathbb{S}^{4}(R)$. The upper bounds of Theorem 1.3 is

$$
4 \kappa_{+}(M)\|H\|_{\infty}\left(4 \kappa_{+}(M)\|H\|_{\infty}+\left(\frac{2}{3} \text { Scal Id }-2 \text { Ric }\right)_{+}\right)=\frac{24}{R^{4}},
$$

which is the first eigenvalue of the Paneitz operator on $\mathbb{S}^{4}(R)$ (see [8]). Hence equality in Theorem 1.3 is attained.

Example 3.3. For the Steklov-Wentzell problem, the same example provide the sharpness of Theorem 1.5. Indeed, for the sphere $\mathbb{S}^{n}(R)$ of radius $R$ into $\mathbb{R}^{n+1}$, the upper bound of Theorem 1.5 is

$$
\kappa_{+}(M)\|H\|_{\infty}\left(\frac{1}{\inf _{M} H}+b n\right)=\frac{R+b n}{R^{2}} .
$$

On the other hand, in 20, Theorem 3.2], we prove that for the sphere

$$
\alpha_{1} V\left(\mathbb{S}^{n}(R)\right)=\frac{1}{R^{2}}\left(n V\left(B^{n+1}(R)\right)+b n V\left(\mathbb{S}^{n}(R)\right)\right) .
$$

An immediate computation using the fact that $V\left(\mathbb{S}^{n}(R)=\omega_{n} R^{2}\right.$ and $V\left(B^{n+1}(R)\right)=\frac{\omega_{n} R^{n+1}}{n+1}$ where $\omega_{n}$ is the volume $\mathbb{S}^{n}$, we obtain that $\alpha_{1}=\frac{R+b n}{R^{2}}$ and equality occurs in Theorem 1.5 .

Example 3.4. Finally, for the biharmonic Steklov problem, we consider again $\mathbb{S}^{n}(R)$ into $\mathbb{R}^{n+1}$. In that case, the first eigenvalue is (see [20, Theorem 3.3])

$$
\beta_{1}=\frac{(n+1) \tau}{R^{2}} \frac{V\left(B^{n+1}(R)\right)}{V\left(\mathbb{S}^{n}(R)\right)}=\frac{\tau}{R}
$$


which coincides with the upper bound of Theorem 1.6 , that is, $\tau \kappa_{+}(M) \frac{\|H\|_{\infty}}{\inf _{M}(H)}$.

\section{REFERENCES}

ACR [1] H. Alencar, M.P. Do Carmo \& H. Rosenberg, On the first eigenvalue of Linearized operator of the $r$-th mean curvature of a hypersurface, Ann. Glob. Anal. Geom., 11 (1993), 387-395.

AM [2] L.J. Alias \& J.M. Malacarne, On the first eigenvalue of the linearized operator of the higher order mean curvature for closed hypersurfaces in space forms, Illinois J. Math. 48 (1) (2004) 219-240.

Ba [3] C. Bär, Extrinsic Bounds for Eigenvalues of the Dirac Operator, Ann. Glob. Anal. Geom. 16(6) (1998), 573-596.

BC [4] J.L.M. Barbosa \& A.G. Colares, Stability of hypersurfaces with constant r-mean curvature, Ann. Global Anal. Geom. 15 (1997), 277-297.

BCP [5] M. Batista, M.P. Cavalcante \& J. Pyo, Some isomperimetric inequalities and eigenvalue estimates in weighted manifolds, J. Math. Anal. Appl., 419(1) (2014), 617-626.

Br [6] T. Branson, Differential Operators Canonically Associated to a Conformal Structure, Math. Scand. 57 (1985), 293-345.

BP [7] D. Buoso \& L. Provenzano, On the eigenvalues of a biharmonic Steklov problem, Integral Methods in Science and Engineering: Theoretical and Computational Advances, Birkhauser, 2015.

CL [8] D. Chen \& H. Li, The sharp estimates for the first eigenvalue of Paneitz operator on 4dimensional submanifolds, arXiv:1010.3102.

DKL [9] M. Dambrine, D. Kateb and J. Lamboley, An extremal eigenvalue problem for the WentzellLaplace operator, Ann. I. H. Poincaré, 33 (2016), no. 2, 409-450.

DHL [10] Z. Djadli, E. Hebey \& M. Ledoux, Paneitz-type operators and applications, Duke Math. J., 104(1) (2000), 129-169

CM [11] M.C. Domingo-Juan \& V. Miquel, Reilly's type inequality for the Laplacian associated to a density related with shrinkers for MCF, arXiv:1503.01332.

EI [12] A. El Soufi \& S. Ilias, Une inégalité de type "Reilly" pour les sous-variétés de l'espace hyperbolique, Comment. Math. Helv. 67(2) (1992), 167-181.

Gi [13] N. Ginoux, Reilly-type spinorial inequalities, Math. Z. 241(3) (2002), 513-525.

Gr [14] J.F. Grosjean, Upper bounds for the first eigenvalue of the Laplacian on compact manifolds, Pac. J. Math. 206(1) (2002), 93-111.

$\mathrm{He}$ [15] E. Heintze, Extrinsic upper bounds for $\lambda_{1}$, Math. Ann. 280 (1988), 389-402.

Pan0 [16] S. Paneitz, A quartic conformally covariant differential operator for arbitrary pseudoRiemannian manifolds, preprint, 1983.

Pan [17] S. Paneitz, A quartic conformally covariant differential operator for arbitrary pseudoRiemannian manifolds, SIGMA 4 (2008), article 036.

$\operatorname{Re}[18]$ R.C. Reilly, On the first eigenvalue of the Laplacian for compact submanifolds of Euclidean space, Comment. Math. Helv. 52 (1977), 525-533.

Ro1 [19] J. Roth, Upper bounds for the first eigenvalue of the Laplacian in terms of anisiotropic mean curvatures, Results Math. 64 (3-4) (2013), pp 383-403.

Ro [20] J. Roth, Reilly-type inequalities for Paneitz and Steklov eigenvalues, Potential Anal. (in press), https://doi.org/10.1007/s1111.

Sa [21] A. Savo, On the first Hodge eigenvalue of isometric immersions, Proc. Amer. Math. Soc. 133 (2005), 587-594.

XW] [22] C. Xia \& Q. Wang, Eigenvalues of the Wentzell-Laplace Operator and of the Fourth Order Steklov Problems, J. Diff. Equations 264(10) (2018), 6486-6506.

Xi [23] C. Xiong, Eigenvalue estimates of Reilly type in product manifolds and eigenvalue comparison for strip domains, Diff. Geom. Appl. 60 (2018), 104-115.

YX [24] P. Yang \& X. Xu, Positivity of Paneitz operators, Discrete Cont. Dyn. Syst. 7(2) (2001), 329-342.

Laboratoire d'Analyse et de Mathématiques Appliquées, UPEM-UPEC, CNRS, F-77454 MARNE-LA-VALLÉE

Email address: julien.roth@u-pem.fr 